\title{
INGLÊS PARA PROPÓSITOS ESPECÍFICOS (IPE): CARACTERÍSTICAS E TENDÊNCIAS ATUAIS DE ENSINO
}

\author{
Stefanie Fernanda Pistoni DELLA ROSA* \\ Cláudia Jotto KAWACHI-FURLAN ** \\ Eliane Hércules AUGUSTO-NAVARRO***
}

- RESUMO: A área de ensino de Inglês para Propósitos Específicos se consolida cada vez mais como uma metodologia de ensino focada e orientada para atender as necessidades específicas de aprendizes que buscam desenvolver competências que os auxiliem a atuar significativamente em contextos profissionais e/ou acadêmicos. Uma de suas vertentes, o Inglês para Propósitos Acadêmicos, confirma-se como uma tendência atual na área de ensino e aprendizagem de línguas devido à sua proposta de focar nas necessidades comunicativas específicas de membros que atuam em contexto acadêmico desenvolvendo pesquisas, divulgando resultados e interagindo com pesquisadores estrangeiros por meio da língua-alvo. O processo de internacionalização das universidades brasileiras, o papel do inglês como língua da ciência e programas de ensino de inglês em contexto acadêmico-universitário (como o Idiomas sem Fronteiras) intensificaram a proposta de cursos para fins específicos, sobretudo para fins acadêmicos, o que configura uma tendência atual de ensino de LI nesse contexto.

- PALAVRAS-CHAVE: Inglês para Propósitos Específicos. Inglês para Propósitos Acadêmicos. Programa Idiomas sem Fronteiras.

\section{Introdução}

A língua inglesa (LI) representa hoje a língua principal para intermediação de relaçóes em diferentes esferas, como: comércio, turismo, entretenimento e ciência, por exemplo, e por isso é reconhecida como Língua Franca (LF). O número de falantes não

\footnotetext{
* IFSP - Instituto Federal de São Paulo. Departamento Núcleo Comum. Hortolândia- SP - Brasil. 13183-250 - stefanie.dellarosa@gmail.com.

** UFES - Universidade Federal do Espírito Santo. Centro de Ciências Humanas e Naturais Departamento de Línguas e Letras. Vitória - ES - Brasil. 29075-910 - claudiakawachi@gmail.com.

*** UFSCAR - Universidade Federal de São Carlos. Centro de Educação e Ciências Humanas Departamento de Letras. São Carlos - SP - Brasil. 13565-905 - eagustonavarro@gmail.com.
} 
nativos de inglês supera o número de falantes nativos e esse dado pode contribuir para a compreensão sobre o lugar que essa língua hoje ocupa.

Devido a esse fato, buscar o aprimoramento ou o desenvolvimento das habilidades em LI pode significar permitir acesso a grupos que tenham a LI como Língua Franca. Portanto, pensar em cursos que possam considerar as características dos diferentes contextos de uso da LI, bem como limitaçóes de tempo e de investimentos financeiros é um dos principais objetivos de cursos de Inglês para Propósitos Específicos (IPE).

Cada contexto possui especificidades que precisam ser consideradas e, por conseguinte, uma análise criteriosa e contínua de necessidades e interesses dos aprendizes, análise da situação-alvo, análise de registros, análise retórica discursiva com foco em gêneros e subgêneros (esta última com contribuição seminal e significativa de SWALES, 1990) precisam ocorrer, a fim de que um curso possa ser desenvolvido para ir ao encontro das expectativas e necessidades dos aprendizes. Além disso, o contexto de desenvolvimento de um curso de Inglês para Propósitos Acadêmicos (IPA) não pode ser desconsiderado.

Conforme apontado por Della Rosa (2013), a habilidade de leitura não representa mais um desafio para pesquisadores brasileiros, como ocorria na década de 80 , quando o IPE ficou conhecido no Brasil, sobretudo por meio dos estudos e propostas de ensino desenvolvido na PUCSP, através do ESP Project. De acordo com Hutchinson e Waters (2006), o foco do Projeto Nacional de Ensino de Inglês Instrumental incidia no trabalho com estratégias de leitura de textos em inglês, sendo as aulas ministradas em português. Ramos (2009) destaca que após a realização de análise das necessidades do público universitário, foi possível identificar que alunos e professores precisavam ler textos acadêmicos em suas áreas que só estavam disponíveis em língua inglesa.

Atualmente, a demanda de pesquisadores inclui as habilidades de produçáo, como a fala e a escrita. Tais habilidades são essenciais para que esses pesquisadores participem ativamente do processo de internacionalização das universidades, seja compartilhando seus trabalhos (artigos publicados ou apresentaçóes em congressos), participando de programas de intercâmbio ou recebendo pesquisadores estrangeiros.

Nesse sentido, o IPA destaca-se na área de ensino-aprendizagem de línguas em âmbito nacional, visto que auxilia os aprendizes a participar desse processo. Com a implantação do Programa Idiomas sem Fronteiras - Inglês ${ }^{1}$ (IsF), essa demanda foi evidenciada. As duas principais açoóes do programa são os exames de proficiência ${ }^{2}$ e as aulas presenciais ofertadas por meio dos Núcleos de Línguas (NucLis). Os NucLis são responsáveis pela oferta de cursos presenciais que atendam a demanda da comunidade

\footnotetext{
1 Programa criado pelo Ministério da Educação em parceria com a Secretaria de Educação Superior e a CAPES em dezembro de 2012. Disponível em: <http://isf.mec.gov.br/ingles/pt-br/o-programa >Acesso em: 1 fev. 2016.

2 Alunos e servidores de universidades públicas podem realizar o exame de proficiência "TOEFL ITP” gratuitamente nos centros aplicadores. A partir de 2016, alunos que participam do PROUNI também podem fazer essa prova.
} 
acadêmica. Portanto, muitos optam pelo desenvolvimento de cursos para fins específicos, sobretudo o IPA, de modo a contemplar a necessidade específica de alunos, professores e funcionários de determinada universidade.

O objetivo deste trabalho é discutir as características do Inglês para Propósitos Específicos, enfatizando as tendências atuais desse ensino por meio da proposta de cursos com foco no desenvolvimento do Inglês para Propósitos Acadêmicos. Para tanto, nos primeiros itens deste artigo, apresentaremos um resumo do surgimento e das características do IPE, bem como a caracterização do IPA por meio da discussão de dados referentes ao desenvolvimento linguístico de pesquisadores brasileiros e da reflexão sobre o inglês como língua internacional da ciência. Além disso, nos itens seguintes, pretendemos discutir como o processo de internacionalização das universidades e a implantação do Programa IsF acentuaram a demanda por cursos de IPA.

\section{Inglês para Propósitos Específicos (IPE): surgimento e características}

Pensar no ensino de Inglês para Propósitos Específicos é pensar em três questôes principais, conforme apontam Hutchinson e Waters (2006): 1- As demandas de um novo mundo após o final da Segunda Guerra Mundial; 2- Uma revolução nos estudos na área de Linguística; 3- $\mathrm{O}$ aprendiz no centro do processo de ensino e aprendizagem.

De maneira sucinta, esses três aspectos podem ser compreendidos, respectivamente, como: 1- uma grande expansão nos campos científico, comercial e tecnológico após o fim da Segunda Guerra Mundial, que exigiu uma língua internacional que pudesse intermediar as relaçôes entre países e, devido ao poder econômico dos Estados Unidos da América naquele período, essa língua era o inglês. Com isso, o aprendizado da língua inglesa (LI) passou a ser motivado por questóes bem definidas. 2- Os estudos relacionados à língua deixaram de ser descritivos, focando em regras gramaticais e passaram a focar no uso real da língua para comunicação. Uma das grandes contribuiçóes foi constatar que o uso que fazemos da língua varia de um contexto para outro e, se esse uso é diferente, deveria ser possível especificar características desses usos e utilizá-los para desenvolver um curso. 3- Rodgers, em 1969, discutiu a importância de considerar o aluno e suas açóes no processo de ensino e aprendizagem, afirmando que focar em seus interesses e necessidades significa motivá-los para um aprendizado eficaz da língua.

Reconhecidamente, a questão da análise de necessidades dos aprendizes está no bojo do surgimento da proposta para o desenvolvimento de cursos de IPE. Em entrevista recente a Salmani Nodoushan (2015, p.114), Johns afirma concordar com todas "as concepçóes duráveis" de IPE propostas em Swales $(1990)^{3}$, particularmente com a necessidade contínua de pesquisa em relação às necessidades dos aprendizes e a situação

\footnotetext{
3 O autor apresenta 5 conceitos de longa duração (duráveis) com relação ao IPE: (a) autenticidade; (b) baseado em pesquisa, (c) língua/texto, (d) necessidade e (e) aprendizado/metodologia.
} 
alvo (de uso da língua). Johns assevera, ainda, que acrescentaria contexto à lista proposta por Swales e reconhece que ele indubitavelmente também o faria no ponto atual em que está em sua carreira.

Robinson (1991) afirma que uma definição precisa e global sobre IPE pode não ser tarefa fácil, pois o que é específico pra uns, pode não ser específico para outros. Entretanto, o que pode ser definido com relação ao IPE são seus tipos de ensino, com focos distintos, sendo eles: ensino de Inglês para Propósitos Ocupacionais (IPO) e ensino de Inglês para Propósitos Acadêmicos.

Augusto-Navarro (2015) afirma que as especificidades possuem uma gradação. Segundo a pesquisadora, essas especificidades podem ser investigadas a partir de informaçóes dadas pelos próprios aprendizes, de documentos basilares para a comunidade discursiva em foco, dos estudos desenvolvidos na área de IPE, entre outros. Essas fontes, juntamente com os instrumentos para coleta de informaçóes, colaboram para que seja possível desenvolver um curso para fins específicos.

A autora cita como exemplo as questōes linguísticas que envolvem as relaçóes com a língua-alvo que podem ser mais explícitas ou menos explicitas, desde diálogos entre passageiros de táxi e motoristas até participação em intercâmbio acadêmico, respectivamente. Não somente as questóes linguísticas são evidenciadas pela autora, mas também as pragmalinguísticas, uma vez que as relaçôes de poder, por exemplo, também precisam ser discutidas em um curso para fim específico. Kasper (1992, p.6) esclarece o sentido do termo pragmalinguística ao afirmar que: "[...] ao exercer determinado ato discursivo os interlocutores escolhem dentre uma variedade de estratégias e formas (linguísticas) aquelas que enunciam um mesmo sentido, mas variam de acordo com a relação entre os interlocutores e a polidez".

Compreendemos que a gradação das especificidades, sugeridas por AugustoNavarro (2015), estão em consonância com as consideráveis mudanças que ocorrem desde o surgimento do IPE. Para Hyland (2006b) a análise de registros, que era a base para o desenvolvimento de materiais, cujo foco era identificar características lexicais e gramaticais, cedeu espaço para uma análise, também, de contexto e interação. $\mathrm{O}$ autor afirma que é preciso compreender o IPE de uma maneira mais ampla, preocupada não apenas com aspetos específicos da língua, mas também com práticas comunicativas.

Para entender a língua e as funçóes que ela desempenha, precisamos compreender como ela é utilizada em contextos particulares, identificando os propósitos e participantes que colaboram integralmente para o desenvolvimento de produtos e processo comunicativos particulares (HYLAND, 2006b, p. 386, tradução nossa) ${ }^{4}$.

\footnotetext{
4 "To understand language and the functions it performs for people, we have to appreciate how it is used within particular contexts, identifying the purposes and participants that are integral to the construction of particular communicative processes and products".
} 
De acordo com Kennedy (2012), a expansão de projetos voltados para o ensino de IPE está diretamente relacionada ao processo de globalização e políticas linguísticas associadas a negócios internacionais, que demanda que os envolvidos nessa área tenham conhecimento de inglês. Além da necessidade evidenciada pelo mercado de trabalho, o autor também destaca que o processo de internacionalização acelerou a implementação do inglês como meio de instrução nas universidades. Segundo Kennedy:

A demanda de alunos pelo ensino em língua inglesa tem feito com que universidades ao redor do mundo reconheçam a necessidade de especialistas falantes de língua inglesa na graduação e pós-graduaçáo assim como para o corpo docente ter acesso ao inglês como língua da academia para publicaçáo e para o desenvolvimento da carreira (KENNEDY, 2012, p. 51-52, tradução nossa ${ }^{5}$ ):

Embora o processo de internacionalização das universidades tenha contribuído para enfatizar a necessidade do uso do inglês como meio de instrução, aulas de cursos de graduação e pós-graduação ministradas em inglês ainda podem representar uma realidade distante no contexto brasileiro. No entanto, a procura por cursos de inglês no ambiente acadêmico tem sido intensificada, o que revela a necessidade de refletirmos acerca da oferta de aulas com foco no IPA. Certamente o investimento em pesquisas e práticas pedagógicas para o ensino-aprendizagem de IPA em contexto nacional é factível e merece atenção.

\section{Inglês como língua internacional da ciência e Inglês para Propósitos Acadêmicos}

Antes de pensarmos nas características do ensino de IPA, é importante discutir sobre o reconhecimento da LI como língua internacional da ciência. Segundo Hyland (2006b), mais de 90\% de periódicos de diferentes áreas de estudo são impressos em inglês. Esse dado pode ilustrar a importância de que a divulgação de ciência para níveis internacionais ocorra nessa língua. Em recente pesquisa Della Rosa (2013) constatou o reconhecimento da LI como língua internacional da ciência por pesquisadores de uma instituição pública brasileira, atribuindo a ela papel fundamental para divulgaçáo de estudos e estabelecimento de parcerias entre pesquisadores.

Devido ao grande número de publicaçóes científicas e conferências realizadas em inglês, é essencial que os pesquisadores desenvolvam a competência na língua para que possam acompanhar os desenvolvimentos na área de atuação deles, assim como ter suas pesquisas disponíveis internacionalmente e conseguirem interagir com

\footnotetext{
5 "Student demand for English-medium education has resulted in universities world-wide acknowledging the necessity for English-speaking subject specialists at undergraduate and postgraduate levels and for staff themselves to have access to English as an academic language for publication and career advancement".
} 
outros pesquisadores (KENNEDY, 2001). Hamp-Lyons (2011) reforça essa discussão ressaltando que o papel da LI tem se tornado cada vez mais forte, o que acaba por diminuir o número de publicaçóes em outras línguas e forçar os pesquisadores a publicar seus estudos em inglês. Em consonância com essa afirmação, Hyland (2006a) afirma que pesquisadores encontram com mais frequência referência aos seus trabalhos quando esses são publicados em LI.

Inglês para Propósitos Acadêmicos pode ser compreendido como o ensino de inglês para auxiliar aprendizes nos estudos ou pesquisa na língua-alvo (FLOWERDEW; PEACKOCK, 2001; JORDAN, 2012). Hyland (2006a) expande essa definição afirmando que além do foco no que pode auxiliar alunos a desenvolverem habilidades que permitam acesso à compreensão e produção científica, também considera IPA a análise de gêneros pertencentes a esse contexto, como defesas orais de doutorado ou documentos de cursos.

O autor ressalta o que IPA "foca, atualmente, em buscar especificidades da língua utilizada na academia [...] incorporando e indo além do contexto comunicativo imediato" (HYLAND, 2006a, p. 2). Podemos depreender, então, que não se trata de um ensino focado apenas nas características de textos ou contextos, mas sim, uma prática que permite atuar significativamente em contextos acadêmicos.

Cursos de IPA possuem a mesma característica principal que os demais cursos de IPE, ou seja, são baseados em uma análise criteriosa de necessidades e, segundo Horowitz (1986), precisam considerar os requisitos acadêmicos e não apenas as particularidades de disciplinas. Robinson (1991) explica que um curso de IPA deve identificar os requisitos sociais e acadêmicos de uma situação específica e capacitar alunos para contribuir nessas situações, ou seja, ela defende a análise cuidadosa da situação alvo no planejamento dos cursos para propósitos específicos.

Com base nessas afirmaçôes, verificamos a importância que se dá ao papel social atribuído à língua nesses contextos de estudo e pesquisa. Por essa razão, ao desenvolver cursos de IPA é imprescindível propiciar aos aprendizes oportunidades de desenvolverem habilidades comunicativas que permitam atuar nesses contextos de maneira sensibilizada. Dessa forma, é importante que aprendizes consigam observar as especificidades deste contexto para que consigam atuar significativamente neles por meio de suas escolhas linguísticas, sua compreensão e análise.

Segundo Liyanage e Birch (2001) o primeiro objetivo de cursos que focam no ensino de IPA é desenvolver nos aprendizes a sensibilização para uma linguagem específica para a atuação no contexto acadêmico. Verificamos na sequência asserçóes de pesquisadores brasileiros a respeito do reconhecimento da LI como língua internacional da ciência, bem como suas justificativas a respeito do interesse em desenvolver habilidades em LI para atuarem nesse contexto. 


\section{Desempenho Acadêmico em LI}

Neste item apresentamos alguns dados obtidos em pesquisa realizada nos anos de 2011 e 2012 cujo objetivo era compreender os interesses e necessidades de pesquisadores de uma universidade pública em desenvolver ou aprimorar o nível de competência linguística em LI para propósitos acadêmicos (DELLA ROSA, 2013). Como instrumentos para coleta de dados foram utilizados dois questionários semiestruturados, os quais foram enviados via e-mail para os pesquisadores (pósgraduandos e docentes) de uma universidade pública localizada no interior do estado de São Paulo. Os participantes foram selecionados mediante o envio das respostas do primeiro questionário, totalizando 205 participantes. O segundo questionário foi enviado aos 199 participantes que afirmaram se interessar por um curso de IPA, obtendo a resposta de 68 participantes.

Neste estudo, ficou evidente o reconhecimento da importância da LI no contexto acadêmico, uma vez que $202(98,5 \%)$ participantes responderam afirmativamente utilizar a língua inglesa em contexto acadêmico justificaram utilizá-la para estar em contato com publicaçóes em periódicos internacionais e com pesquisadores estrangeiros, além de reconhecê-la como a língua necessária para publicação, entre outros, conforme verificamos nas asserçóes a seguir:

M5: Pelo menos em minha área de estudos, praticamente todos os periódicos publicam seus textos em inglês. Logo, ler e escrever de maneira correta nesta língua é fundamental para a carreira. (Diversidade Biológica e Conservação)

D3: $\mathrm{O}$ conhecimento e a compreensão da língua inglesa são essenciais para todas as pessoas, já que é a língua mais difundida no mundo. No meio acadêmico o uso da língua inglesa é utilizada diariamente tanto em rótulos de produtos e reagentes, quanto em manuais, artigos, contatos, em congressos ou encontros, viagens e etc. Nos dias de hoje, um bom profissional tem a necessidade de falar, entender e escrever em inglês. (Biologia molecular e conservação)

P6: Pelas atuais configuraçôes do sistema de qualificação da produção científica, assim como pelos incentivos à internacionalização da pesquisa, faz-se fundamental a comunicação com a língua inglesa. (Terapia Ocupacional) ${ }^{6}$

Segundo Mauranen (2011, p. 94) "a língua inglesa se tornou a língua franca da academia, assim como de outros contextos”. Em relação à internacionalização, conforme afirmado por uma das participantes, Long e Richards (2001) ponderam que a LI é a língua necessária para a disseminação de pesquisa nas áreas de ciência, tecnologia e indústria.

${ }_{6}$ As iniciais M, D e P referem-se, respectivamente, a mestrandos, doutorandos e professores. 
Em virtude do reconhecimento da LI como língua internacional da ciência, é fundamental que pesquisadores desenvolvam suas habilidades em LI a fim de que possam participar ativamente do ambiente acadêmico, seja divulgando suas pesquisas em congressos ou periódicos internacionais. Os participantes da pesquisa em questão reconhecem essa necessidade e 199 (97\%) responderam afirmativamente à pergunta Você considera importante aprimorar seus conhecimentos acerca da lingua inglesa tendo em vista sua vida acadêmico-profissional? Justifique sua resposta.

M7: As pesquisas de ponta e com reconhecimento internacional são todas em inglês.

Considero impossível manter-se no meio acadêmico sem domínio desta língua. (Enfermagem)

M24: O inglês é a linguagem universal da ciência. É muito ruim perceber que seu desempenho, em grande parte, é comprometido pela não fluência no idioma. (Ciência da Computação)

D4: A língua inglesa como sabemos é uma língua universal e a mais utilizada no meio acadêmico. Sem o conhecimento da língua, nosso trabalho e a repercussão dele dentro da área de pesquisa se tornam limitada. (Biotecnologia)

Para Kennedy (2001), é fundamental que pesquisadores desenvolvam a competência na língua para que possam acompanhar os desenvolvimentos em sua área de atuação, bem como disponibilizar suas pesquisas internacionalmente e poderem interagir com pesquisadores estrangeiros. Os autores Cargill, O'Connor e Li (2012), reiteram essa afirmação ressaltando a importância de desenvolver, em conjunto com os conhecimentos científicos, as habilidades em LI para que possam divulgar seus estudos internacionalmente.

Apesar do reconhecimento, por parte de pesquisadores, 1) da LI como língua internacional da ciência e 2) da necessidade em desenvolver habilidades comunicativas em LI para atuar em contextos acadêmicos, ao serem questionados sobre o que deveria ser feito para auxiliar o pesquisador na divulgação de pesquisas, os participantes sugeriram que as universidades deveriam ser uma das principais responsáveis em oportunizar possibilidades de ensino de LI para fins acadêmicos, como é possível verificar nas seguintes afirmaçóes:

M33: Acredito que as Universidades poderiam criar um programa para os alunos de graduação que queiram ingressar na carreira acadêmica, para o aprofundamento de como funciona o processo de comunicação científica. (Ciência, Tecnologia e Sociedade).

D2: Seria muito interessante que os cursos de graduação oferecessem uma disciplina, ou mais que uma disciplina, um acompanhamento ao longo do curso para escrita científica em inglês. [...] Se tivesse essa disciplina no segundo ano de curso (período em que os alunos já adquiriram uma certa maturidade acadêmica e que ainda há 
tempo para se aprender) e depois houvesse uma espécie de monitoria, para que alunos interessados continuassem seu aprendizado, seria muito interessante e produtivo. (Ecologia)

P6: Na universidade, eu acho que, talvez, pudesse haver algum estímulo um pouco diferenciado para a cultura da língua inglesa, por exemplo, hoje vemos isso no programa Ciências sem Fronteiras. Em muitos casos a barreira que existe é a barreira da língua. É necessário eliminar essa barreira da língua desde que ele inicia a graduação. Mas ações de popularizar a língua é importante. (Química)

Com essas afirmações podemos verificar a importância que os participantes atribuem ao aprendizado da língua inglesa desde a graduação, devido à sua importância na área de desenvolvimento de pesquisa. Compreendemos que o principal foco de um curso para propósitos específicos e acadêmicos é a análise de necessidades e neste caso, podemos entender a Análise da Situação-Alvo (Target Situation Analysis) como sendo uma das principais análises a serem realizadas para o desenvolvimento de cursos de IPA, uma vez que ela verifica as necessidades linguísticas existentes em contextos específicos (WEST, 1994, citado por RAHMAN, 2015).

Tendo em vista a importância da análise da situação-alvo, muitos NucLis de universidades federais brasileiras propóem cursos voltados para a escrita acadêmica em língua inglesa, visando a atender a demanda de sua comunidade acadêmica, conforme discutido no item a seguir.

\section{IPE e IPA nas universidades federais brasileiras}

De acordo com Finardi e Porcino (2014, p. 241), a globalização contribuiu para a internacionalização das universidades, evidenciando a necessidade de comunicação da sociedade globalizada. Knight (2004) reflete sobre a dificuldade em definir internacionalização devido aos diferentes sentidos atribuídos a essa palavra, além das confusóes associadas ao termo. A autora propóe uma definição visando a abordar os diferentes níveis e a relação dinâmica entre eles: "[...] internacionalização no nível nacional, setorial e institucional é definida como o processo de integrar uma dimensão internacional, intercultural e global ao propósito, função e oferta de educação de nível superior $^{7 "}$ (KNIGHT, 2003, p. 2, tradução nossa).

Nesse sentido, ao considerar o processo de internacionalização das universidades brasileiras, é necessário pensar a educação em nível superior como internacional, intercultural e global. Para tanto, a comunicação em língua inglesa torna-se essencial para que a comunidade acadêmica participe desse processo. Finardi e Archanjo (2015),

\footnotetext{
"Internationalization at the national, sector, and institutional levels is defined as the process of integrating an international, intercultural, or global dimension into the purpose, functions or delivery of postsecondary education".
} 
com base na análise de programas de internacionalização no Brasil, afirmam que o inglês é visto como língua estrangeira na educação básica, sendo enfatizado o desenvolvimento da habilidade de leitura. Porém, na educação superior, o inglês possui o status de língua internacional.

Tendo em vista a importância da LI nas esferas profissionais, acadêmicas, econômicas, e, recentemente, sua função central nos processos de internacionalização das instituições de ensino superior, uma vertente desse idioma que recebe atenção é o Inglês para Propósitos Acadêmicos (IPA). Conforme defendido por Hyland e Hamp-Lyons (2002), o IPA enfatiza o desenvolvimento das habilidades do aprendiz para que ele participe ativamente de uma comunidade específica. Assim, o ensino de IPA pode contribuir para auxiliar pesquisadores a divulgarem os resultados de suas pesquisas em inglês, participando, assim, ativamente de sua comunidade discursiva, como assevera Della Rosa (2013). Dessa forma, o ensino de IPA parece apropriado para esse público, visto que, conforme afirmam Dudley-Evans e St. John (2010), o Inglês para Propósitos Acadêmicos privilegia o ensino de LI relacionado a um propósito acadêmico.

Esse propósito acadêmico tem sido foco de cursos de inglês oferecidos por meio do Programa Idiomas sem Fronteiras. Como apresentando anteriormente, o programa foi implantando no final de 2012. No momento de sua criaçáo, o objetivo do programa era auxiliar o Ciência sem Fronteiras (CsF). Pinheiro e Finardi (2014) esclarecem que no início do CsF, houve pouca procura de candidatos para bolsas de estudos no exterior, levantando a suspeita de que os possíveis candidatos apresentavam dificuldades em realizar exames de proficiência. Na tentativa de minimizar tais dificuldades, houve a criação do Programa Inglês sem Fronteiras (IsF) cujo objetivo, no início, era cadastrar universidades federais como Centro Aplicadores (CA) do exame TOEFL ITP. As autoras destacam que, embora a adesão ao programa tivesse melhorado com essa ação, ainda não havia candidatos suficientes para as vagas do CsF. Nesse momento, a dificuldade identificada era com relação à pontuação necessária no exame de proficiência.

Com o intuito de auxiliar os candidatos a melhorar a proficiência linguística, houve a implantaçáo dos Núcleos de Línguas (NucLi). Pinheiro e Finardi (2014, p. 76) apontam que "[...] o objetivo dos núcleos criados é ofertar aulas presenciais e apoio online aos candidatos ao programa CsF para que eles possam desenvolver sua competência linguística para passar na prova do TOEFL”. Atualmente, o programa Inglês sem Fronteiras expandiu para outras línguas e, por isso, passou a ser denominado Idiomas sem Fronteiras. $\mathrm{O}$ objetivo do programa também ampliou, visto que náo objetiva apenas preparar alunos para serem aprovados em exames de proficiência, uma vez que o intuito do programa é:

O Idiomas sem Fronteiras conta atualmente com 63 núcleos em universidades federais para ofertar cursos presenciais de inglês. Os cursos visam enfatizar o desenvolvimento da comunicação oral e escrita, o conhecimento de culturas 
acadêmicas em países onde se usa a língua inglesa no ensino superior e a interação aluno-aluno e professor-aluno ${ }^{8}$.

Percebemos, assim, que as aulas presenciais do IsF visam a promover o desenvolvimento da habilidade oral e escrita, bem como a sensibilização cultural para uso da língua no âmbito acadêmico. Nesse sentido, o ensino de Inglês para Propósitos Específicos ganha destaque nesse contexto, visto que os NucLis passam a oferecer cursos presenciais que atendam a necessidades específicas de sua comunidade.

Conforme discutido por Augusto-Navarro (2015), há uma gradação na especificidade das necessidades. Portanto, diversos cursos foram criados considerando a necessidade do público-alvo de cada universidade. Como cada NucLi tem liberdade e flexibilidade para elaborar o programa de seus cursos (desde que estejam de acordo com as orientaçóes do Núcleo Gestor - equipe que gerencia o IsF), os cursos podem ter foco, por exemplo, até em conversas telefônicas, ou seja, atendendo as necessidades específicas de funcionários de secretaria que precisam do inglês para essa situação. Por outro lado, como a pesquisadora pondera, há necessidades que não são transparentemente especificáveis, como o caso de cursos que buscam sensibilizar alunos que participarão de intercâmbio acadêmico.

Nesse contexto, a procura por cursos de Inglês para Propósitos Acadêmicos foi acentuada. Com o processo de internacionalização e a necessidade de pesquisadores publicarem seus trabalhos, seja por meio da publicação de seus artigos ou pela participaçâo em congressos e jornadas científicas, há a demanda por cursos que visem a atender essa necessidade. Logo, podemos ressaltar que a internacionalização, a constatação do papel do inglês como língua internacional do ensino superior (FINARDI; ARCHANJO, 2015) e a implantação do programa IsF contribuíram para retomada, nacionalmente, de cursos para fins específicos, sobretudo cursos de IPA, apresentando-se como tendência atual no ensino de línguas.

\section{Conclusão}

Diante do cenário de consolidação da língua inglesa como língua não apenas da ciência, mas também como língua global (CRYSTAL, 2003) e de pesquisadores buscando aprimoramento de suas habilidades para produção científica, a procura por cursos de Inglês para Propósitos Acadêmicos tem sido evidenciada. Tal procura pode ser observada nas propostas de cursos ofertados por meio do Programa Idiomas sem Fronteiras. Destacamos que cursos de IPA têm como principal característica preparar aprendizes para atuarem significativamente em contexto de estudo e pesquisa, seja publicando seus resultados de pesquisa, interagindo com demais aprendizes e pesquisadores ou atualizando seus conhecimentos.

8 Disponível em: <http://isf.mec.gov.br/ingles/curso-presencial>. Acesso em: 25 fev. 2016. 
Com base no reconhecimento da necessidade da língua inglesa para o processo de internacionalização das universidades brasileiras, do papel do inglês como língua internacional na educação superior (FINARDI; ARCHANJO, 2015) e ações governamentais (como CsF e o IsF), observamos a importância de se pensar na oferta de cursos de Inglês para Propósitos Específicos, principalmente cursos voltados para atender a demanda da comunidade acadêmica, enfatizando a oferta de cursos para fins acadêmicos.

Por fim, destacamos a fala de Johns em entrevista concedida a Salmani Nodoushan (2015, p.118, tradução nossa): "Estou envolvida com o planejamento de cursos e pesquisa em IPE há mais de 30 anos e continuo a aprender e a revisar meus pensamentos. Especialistas em IPE devem ser abertos, flexíveis, sensíveis ao contexto - e ótimos aprendizes!" 9

Percebemos, assim, a importância de dedicarmos atenção à questão do IPA em pesquisas, práticas pedagógicas e formação de professores. É uma área de campo fértil e dinâmico e que tem muito ainda a ser explorado e gerado como frutos rumo a um maior empoderamento de pesquisadores brasileiros.

\section{ENGLISH FOR SPECIFIC PURPOSES (ESP): CHARACTERISTICS AND CURRENT TENDENCIES IN LANGUAGE TEACHING}

- ABSTRACT: The teaching area of English for Specific Purposes has consolidated itself as a teaching approach focused and oriented to attend specific learners' needs interested in developing competences that help them to participate in occupational andlor academic contexts meaningfully. One of its branches, English for Academic Purposes, can be considered a current tendency in language teaching due to its aim at focusing on specific communicative needs of members that participate in the academic contexts either conducting research and publishing results or interacting with foreign researchers through the target language. The internalization process of Brazilian universities, the role of English as the language of science and English teaching programs at universities (such as Language without Borders) has intensified the proposal of courses for specific purposes, especially for academic purposes, which categorizes itself as a current tendency in English teaching contexts.

- KEYWORDS: English for Specific Purposes. English for Academic Purposes. Language without Borders.

\footnotetext{
9 "I have been involved in ESP research and curriculum development for more than 30 years, and I continue to learn and revise my thinking. ESP specialists must be open, flexible, sensitive to context-and very good learners!"
} 


\section{REFERÊNCIAS}

AUGUSTO-NAVARRO, E. H. Análise de Necessidades/Interesses no ELFE: foco na gradação da especificidade. 2015. Disponível em: <https://www.youtube.com/watch?v=NFKX MM8bJUg\&feature=youtu.be $>$. Acesso em: 21 set. 2015.

CARGILL, M.; O'CONNOR, P.; LI Y. Educating Chinese scientists to write for international journals: Addressing the divide between science and technology education and English language teaching. English for Specific Purposes, New York, v.31, n.1, p.60-69, 2012.

CRYSTAL, D. English as a Global Language. New York: Cambridge University Press, 2003.

DELLA ROSA, S. Análise das reflexóes estabelecidas por pesquisadores entre conhecimento de língua inglesa e desempenho acadêmico. 2013. 148f. Dissertação (Mestrado em Linguística) - Centro de Educação e Ciências Humanas, Universidade Federal de São Carlos, São Carlos, 2013.

DUDLEY-EVANS, T.; ST. JOHN, M. Developments in English for Specific Purposes: A multidisciplinary approach. 12ed. Cambridge: Cambridge University Press, 2010.

FINARDI, K. R.; PORCINO, M. C. Tecnologia e Metodologia no Ensino de Inglês: Impactos da Globalização e da Internacionalização. Revista Ilha do Desterro, Florianópolis, n.66, p.239-282, jan/jun. 2014.

FINARDI, K. R.; ARCHANJO, R. Reflections of Internationalization of Education in Brazil. International Business and Education Conference Proceedings, New York, v.1, p.504-510, 2015.

FLOWERDEW, J.; PEACOCK, M. Issues in EAP: A preliminary perspective. In: FLOWERDEW, J.; PEACOCK M. (Org.). Research perspectives on English for Academic Purposes. Cambridge: Cambridge University Press, 2001. p.177-194.

HAMP-LYONS, L. English for Academic Purposes: 2011 and beyond. Journal of English for Academic Purposes, Oxford, v.10, n.1, p.1-3, 2011. Available at: <http://www.sciencedirect. com/science/journal/14751585/10/1>. Access on: 9 set. 2016.

HOROWITZ, D. Essay examination prompts and the teaching of academic writing. English for Specific Purposes, Oxford, v.5, n.2, p.107-120, 1986.

HUTCHINSON, T.; WATERS, A. English for Specific Purposes: A learning-centred approach. New York: Cambridge University Press, 2006.

HYLAND, K. English for academic purposes: an advanced resource book. New York: Routledge, 2006a.

HYLAND, K. English for specific purposes: some influences and impacts. In: CUMMINS, J.; DAVISON, C. (Org.). The International Handbook of English language education. 
v.1. Norwell, Mass: Springer, 2006b. p. 379-390. Available at: <http://www2.caes.hku.hk/ kenhyland/files/2012/08/English-for-specific-purposes_some-influences-and-impacts.pdf>. Access on: 21 sept. 2016.

HYLAND, K.; HAMP-LYONS, L. EAP: issues and directions. Journal of English for Academic Purposes, Oxford, v.1, n.1, p.1-12, 2002. Available at: <whttp://www.sciencedirect. com/science/journal/14751585/1/1>. Access on: 9 sept. 2016.

JORDAN, R. R. English for Academic Purposes: A guide and resource book for teachers. 13.ed. Cambridge: Cambridge University Press, 2012.

KASPER, G. Pragmatic Transfer. University of Hawaii Working Papers in ESL, Honolulu, v.11, n.1, p.1-34, 1992.

KENNEDY, C. ESP Projects, English as a global language, and the challenge of change. Ibérica, Castello de la Plana, n.24, p.43-54, 2012. Disponível em: <http://www.aelfe.org/ documents/05_24_Kennedy.pdf>. Acesso em: 21 set. 2016.

KENNEDY, C. Language use, language planning and EAP. In: FLOWERDEW, J.; PEACOCK, M. (Org.). Research perspectives on English for Academic Purposes. Cambridge: Cambridge University Press, 2001. p.25-41.

KNIGHT, J. Internationalization remodeled: definition, approaches, and rationales. Jornal of Studies in International Education, Thousand Oaks, v.8, n.1, p.5-31, 2004.

KNIGHT, J. Updated internationalization definition. International Higher Education, Dagenham, Essex, v.33, p.2-3, 2003.

LIYANAGE, I.; BIRCH, G. English for general academic purposes: catering to disciplinespecific needs. Queensland Journal of Educational Research, Brisbane, v.17, n.1, p.48-67, 2001.

LONG, M.; RICHARDS, J. Prefácio. In: FLOWERDEW, J.; PEACOCK, M. (Org.). Research Perspectives on English for Academic Purposes. Cambridge: Cambridge University Press, 2001, p. XIII-XIV.

MAURANEN, A. English as the Lingua Franca of the Academic World. In: JOHNS, A.; PAlTRIDGE, B.; BELCHER, D. (Org.). New Directions in English for Specific Purposes Research. Ann Arbor: Michigan University Press, 2011. p.94-117.

PINHEIRO, L.; FINARDI, K. R. Políticas públicas de internacionalização e o papel do inglês: evidências dos Programas CSF e ISF. In: CONEL: CONGRESSO NACIONAL DE ESTUDOS LINGUÍSTICOS, 2., 2014, Vitória. Anais... Vitória: Programa de Pós-Graduação em Linguística, Universidade Federal do Espírito Santo, 2014. p.76-78.

RAHMAN, M. English for Specific Purposes (ESP): A Holistic Review. Universal Journal of Educational Research, Alhambra, v.3, n.1, p.24-31, 2015. 
RAMOS, R. ESP in Brazil: history, new trends and challenges. In: KRZANOWSKI, M. (Org.). English for Academic and Specific Purposes in Developing, Emerging and Least Developed Countries. Reading, UK: Garnet Publishing, 2009. p.68-84.

ROBINSON, P. ESP Today: A Practitioner's Guide. Hempstead, UK: Prentice Hall, 1991.

SALMANI NODOUSHAN, M. A. English for Specific Purposes: The state of the art (An online interview with Ann M. Johns). International Journal of Language Studies, Ipswich, v.9, n.2, p.113-120, 2015.

SWALES, J. M. Genre analysis: English in academic and research settings. Cambridge: Cambridge University Press, 1990.

Recebido em fevereiro de 2016

Aprovado em junho de 2016 
